\title{
Comparison of Effectiveness of Diode Laser as an Adjunct to Scaling and Root Planing and Scaling, Root Planing Alone in Treatment of Chronic Periodontitis-A Randomized Split- mouth Clinical Study
}

\author{
Manjunath Pooja ${ }^{1}$, Rajesh S Kashyap ${ }^{2}$, Shashikanth Hegde ${ }^{3}$, Maiya A Kumar ${ }^{4}$, Vinita Boloor ${ }^{5}$
}

\begin{abstract}
Aim and objective: To compare the clinical effects of diode laser (DL) as an adjunct to scaling and root planing (SRP) and SRP alone in the treatment of subjects with chronic periodontitis.

Materials and methods: Thirty subjects aged 25-54 years participated in this randomized split-mouth clinical study. All subjects with 60 selected sites were randomly divided into 2 equal groups depending on the treatment provided. Group I (control site): only SRP was performed at baseline and group II (test site): SRP along with DL irradiation was performed on day 7, 14, and 21. Clinical parameters plaque index (PI), gingival index $(\mathrm{Gl})$, bleeding index (BI), probing pocket depth (PPD), and clinical attachment level (CAL) were recorded at baseline and the end of 3 months for both groups.

Results: Mean values of PPD from baseline to 3 months between groups I and II on the mesial and distal aspect showed a high statistically significant difference $(p<0.02)$. Mean values of CAL from baseline to 3 months between two groups on distal and buccal aspect showed high statistically significant difference $(p<0.02)$. On intragroup comparison, there was a decrease in a mean difference of $\mathrm{Pl}, \mathrm{Gl}$, and $\mathrm{Bl}$ individually for both the groups from baseline to 3 months with $(p<0.001)$.

Conclusion: The adjunctive use of $810 \mathrm{~nm}$ DL with SRP provides moderate additional clinical benefit in moderate periodontal pockets 4-6 mm. Clinical significance: This study signifies that using noninvasive laser therapy, potential clinical benefits can be obtained in terms of reduction of periodontal parameters.

Keywords: Adult patient, Chronic periodontitis, Dental plaque, Flapless, Gingivitis, Intraoral, Randomized clinical study, Root planing, Ultrasonic scaling.

Journal of Contemporary Dentistry (2019): 10.5005/jp-journals-10031-1275
\end{abstract}

\section{INTRODUCTION}

Chronic periodontitis (CP) is an inflammatory periodontal disease that is most prevalent, affecting the world's population which accounts for $10 \%$. A pathogenic consortium of bacteria surrounding the teeth starts up the process of disease initiation, as a result of this, the immuneinflammatory response leads to the occurrence and progression of the disease from gingivitis to periodontitis in susceptible people. Periodontitis is a chronic inflammatory disease of periodontium, which is of multifactorial origin that is caused due to the accumulation of plaque biofilm and calculus on the root surface of the tooth, which leads to continuous destruction of the periodontal ligament and alveolar bone, along with the formation of pocket, recession, or both. ${ }^{1}$

Production of inflammatory cytokines in excess, especially interleukins (ILs) and tumor necrosis factor-a (TNF-a) are directly related to destroying periodontal tissues. Gingivitis progresses into periodontitis if left untreated. Hence, mechanical plaque control is a mode of therapeutic approach in halting the progression of gingivitis to periodontitis. ${ }^{2}$

Plaque removal performed using manual and powered instruments has shown effective removal of plaque biofilm and calculus from the root surfaces, thereby the bacterial load in periodontal pockets will be reduced. ${ }^{3}$

Complete removal of subgingival plaque biofilm and calculus is not possible while carrying out the instrumentation in deeper
${ }^{1-5}$ Department of Periodontics, Yenepoya Dental College, Mangaluru, Karnataka, India

Corresponding Author: Manjunath Pooja, Department of Periodontics, Yenepoya Dental College, Mangaluru, Karnataka, India, Phone: +91 9686626929, e-mail: poojamysore1@gmail.com

How to cite this article: Pooja M, Kashyap RS, Hegde S, et al. Comparison of Effectiveness of Diode Laser as an Adjunct to Scaling and Root Planing and Scaling, Root Planing Alone in Treatment of Chronic Periodontitis-A Randomized Split-mouth Clinical Study. J Contemp Dent 2019;9(3):117-123.

Source of support: Nil

Conflict of interest: None

periodontal pockets and furcations, because it is doubtful whether they can reach the surfaces of roots of the teeth.

For these various reasons, different local or systemic antibiotics are used to treat periodontitis. Furthermore, it will be very difficult to maintain these antibiotics at a concentration which gives therapeutic benefits in terms of reduction of the periodontal pocket, and also there is an enormous concern regarding the development of antibiotic resistance. ${ }^{6}$

Because of this aspect, and also the use of laser radiation within the periodontal pocket causes sulcus disinfection (antibacterial), 
photostimulatory effect, the laser has gained much interest and its been promising therapeutic modality in the specialty of periodontology. As conventional mechanical instrumentation cannot reach certain sites, the intended therapeutic benefit cannot be achieved but the same can be achieved by lasers, as they cause good tissue ablation, bactericidal and detoxification effects. ${ }^{4}$ The laser wavelengths most commonly used in the field of periodontology include erbium:yttrium-aluminum-garnet (Er:YAG) lasers, diode lasers (DLs), neodymium:yttrium-aluminum-garnet ( $\mathrm{Nd}: \mathrm{YAG})$, and the carbon dioxide $\left(\mathrm{CO}_{2}\right)$ laser. ${ }^{4}$

In 1995, FDA approved the use of a DL [gallium-aluminumarsenide (Ga-Al-As; $810 \mathrm{~nm}$ )] for oral soft tissue surgery and in 1998 for sulcular debridement. A DL is a semiconductor laser that converts electrical energy into light energy, they have high electrical to optical efficiency, are small lightweight, and compact, hence portable as compared to other solid-state and gas lasers. ${ }^{4}$

Application of DL with a wavelength between 655 and $980 \mathrm{~nm}$ is known to accelerate wound healing bringing about angiogenesis, through the facilitation of formation of collagen, and augmentation of growth factor. ${ }^{5}$ Hence, these cumulative effects of DL promote healing of periodontal tissues, which necessitates the same of the same to obtain the intended therapeutic benefits.

With consideration of the above advantages of DL in causing sulcus disinfection and photostimulatory effects, its use as an adjunct to the scaling and root planing (SRP) can be the choice of treatment in treating $\mathrm{CP}$ patients.

Therefore, the purpose of this study is to compare the clinical effects of DL as an adjunct to SRP and SRP alone in the treatment of CP patients.

\section{Materials and Methods}

This study was a randomized, single-blinded, controlled, parallelgroup clinical study. It was conducted at Yenepoya Dental College and Hospital, Department of Periodontics, Mangaluru from November 2018 to August 2019. The clinical trial approval was given by the Institutional Ethics Committee (2017/292 dated 27/11/2017). Informed consent was obtained from each of the subjects voluntarily, after providing them with a detailed information brochure regarding the study. After receiving the informed consent, we adhered to the principles of the Helsinki declaration that are outlined when human subjects are involved.

\section{Sample Size}

The sample size was calculated for an alpha error of 0.05 and a power of $80 \%$.

\section{Participants}

Thirty systemically healthy subjects aged 25-54 years, suffering from CP were included in the study. The following were the inclusion criteria: untreated subjects in the age group of 25-54 years suffering from generalized CP; minimum of 16 teeth to be present in every subject; subjects with 2 posterior sites with a pocket depth of 4-6 mm; subjects who are negative for any systemic diseases; subjects who have undergone periodontal surgical or non-surgical treatment before 6-12 months of the study; subjects who have received antibiotic regimen and/or anti-inflammatory drugs within 3-6 months before the study, pregnant and lactating mothers; and subjects with the habit of smoking were excluded from the study. Randomized sampling was done in this study, the random allocation of subjects was done using the coin toss method.

\section{Armamentarium}

- Mouth mirror, William's periodontal probe, explorer, tweezer, gloves, mouth mask, disposable syringes, betadine solution, normal saline, local anesthesia ( $2 \%$ lignocaine hydrochloride with 1:80,000 adrenaline) (Fig. 1).

- Gracey curettes No. 1-14 (Hu-Friedy, USA) (Fig. 2).

- Ultrasonic scaler unit (piezo-electric with frequency ranging between 29 and $50 \mathrm{kHz}$ ) (Fig. 3).

- Soft-tissue DL unit (Zolar Technology and Mfg. Co. Inc.) Photon model [optic fiber with a thickness of $400 \mu \mathrm{m}, 2.5 \mathrm{~W}$, a wavelength of $810 \mathrm{~nm}$, input (vol)-DC 16.8 V] (Fig. 4).

\section{Study Design}

This is a randomized, single-blinded, controlled, parallel clinical study. The clinical study was divided into pre-screening, treatment, and posttreatment phases.

\section{Screening Phase}

The subjects indicated for SRP alone (group I) and DL as an adjunct to SRP (group II) were recruited for the study. The importance of maintaining oral hygiene was reinforced by the same operator. Those subjects who fulfilled the inclusion criteria were randomly selected by using the coin toss method.

\section{Treatment Phase}

At baseline, the recruited subjects belonging to both the groups were assessed for periodontal disease in terms of plaque index $(\mathrm{PI})$, gingival index $(\mathrm{GI})$, bleeding index $(\mathrm{BI})$, probing pocket depth (PPD), and clinical attachment level (CAL) and standard oral hygiene instructions were given. Scaling and root planing was done by the examiner on all the subjects with control and test sites (teeth) by using a combination of an ultrasonic scaler (piezo-electric with frequency ranging between 29 and $50 \mathrm{kHz}$ ) with constant water irrigation according to the instructions of the manufacturer and Gracey curettes (Figs 2 and 3).

On day 7, 14, and 21 after SRP, the method of laser irradiation onto the selected sites was done in the following manner:

- The soft tissue DL with a diameter of optic fiber being $400 \mu \mathrm{m}$, wavelength $810 \mathrm{~nm}$, output power $2.5 \mathrm{~W}$, input (vol)-16.8 V, continuous wave mode was used to perform the laser irradiation to achieve better healing and also biostimulation (Fig. 4).

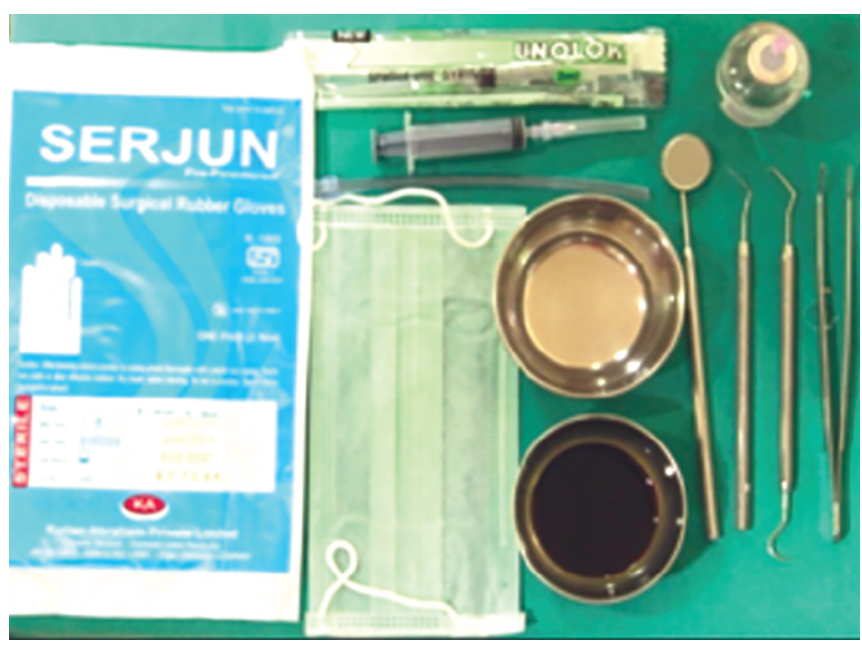

Fig. 1: Armamentarium 


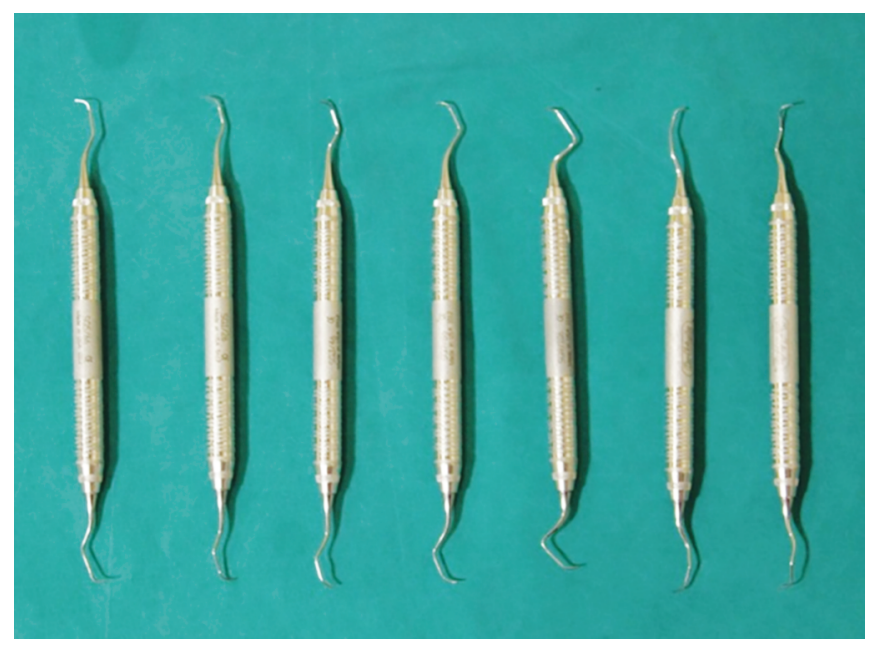

Fig. 2: Gracey curette (No. 1-14)

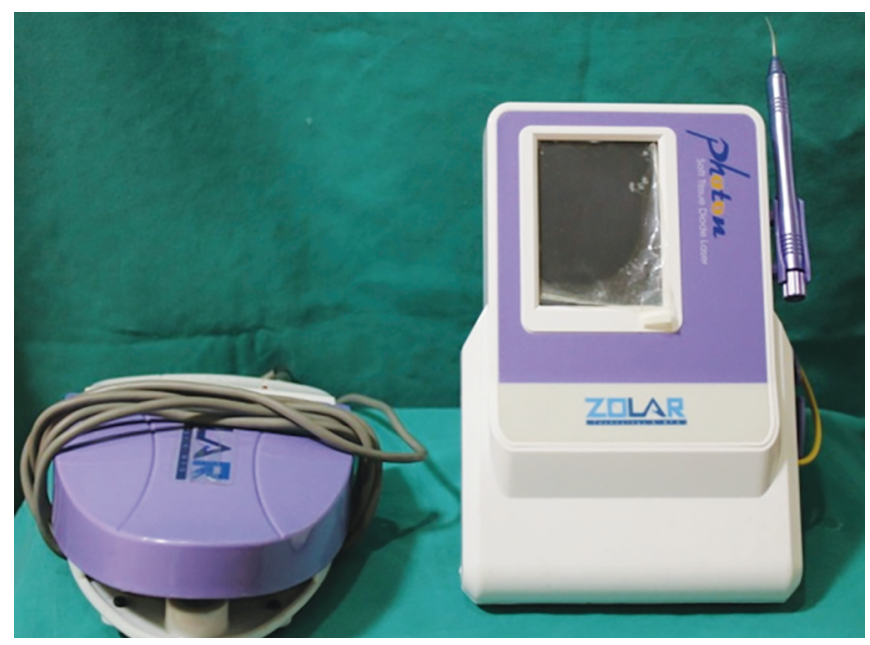

Fig. 4: Soft tissue diode laser unit (Zolar Technology and Mfg. Co. Inc., 6315 Shawson Dr \# 7, Mississauga, ON L5T, Canada)

- The laser optic fiber was measured to a distance of $1 \mathrm{~mm}$ short of the depth of the pocket. The test site was anesthetized by giving infiltration anesthesia by using $2 \%$ lignocaine and 1:80,000 adrenaline before the laser irradiation.

- Along with the continuous irrigation of normal saline, the optic fiber was introduced into the pocket with light contact with apico-coronal motion, such that it covers the full length of the epithelial lining, starting from the base of the pocket and moving upward (apico-coronal direction) for 20 seconds. The fiber tip was cleaned with damp gauze to prevent the debris build-up (Fig. 5) and standard oral hygiene instructions were reinforced to the subjects with test sites and there was no need for postoperative medicaments to be advised to any of the subjects as it was a flapless and noninvasive procedure.

\section{Posttreatment Phase}

On day 90 after SRP, the subjects with both control and the test sites were recalled for the reassessment of the same clinical parameters which was done by a calibrated examiner who was not a part of the study.

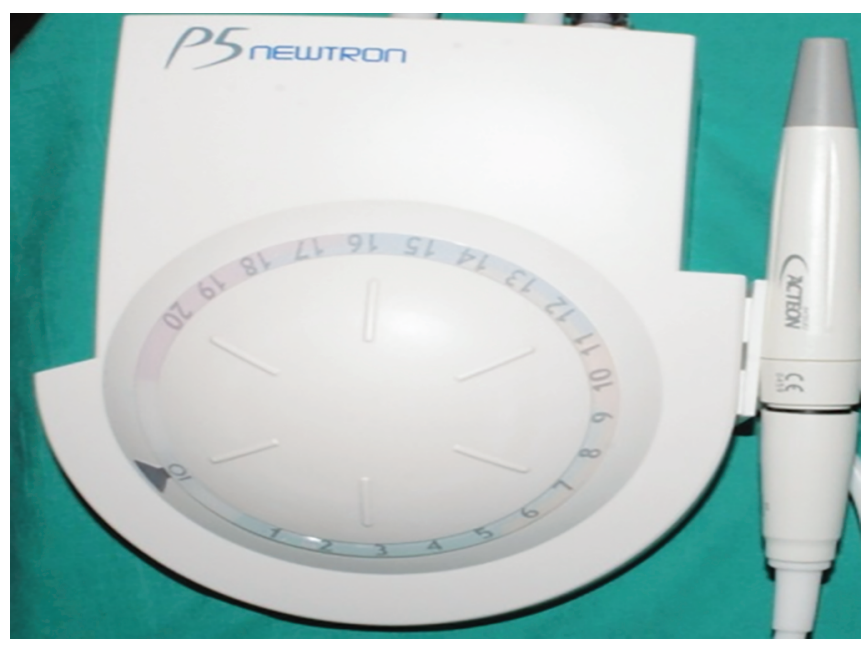

Fig. 3: Ultrasonic scaler unit (piezo-electric with frequency ranging between $29 \mathrm{kHz}$ and $50 \mathrm{kHz}$ )

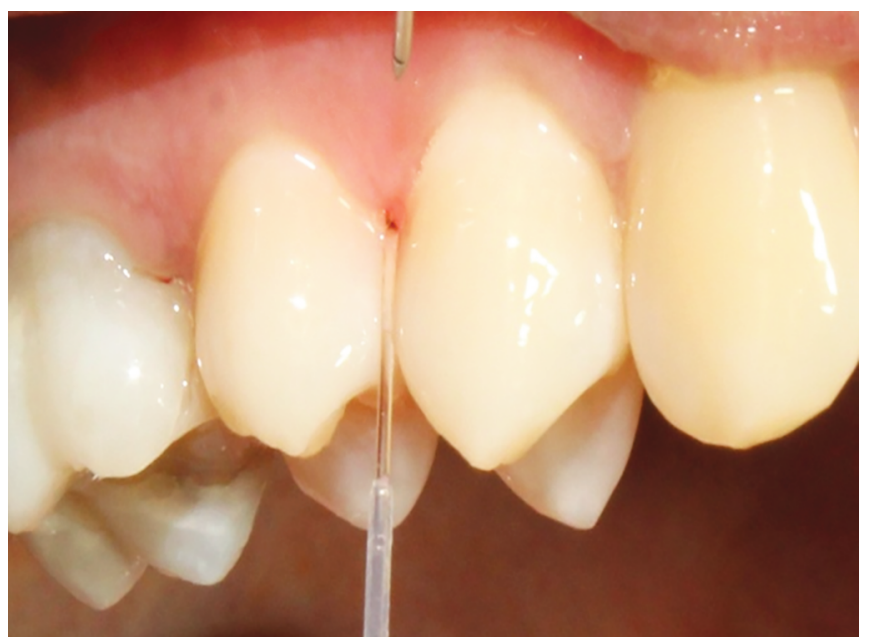

Fig. 5: Laser irradiation (apico-coronal movement of laser tip with copious irrigation of saline using syringe)

\section{Outcome Measurements}

The principal investigator and calibrated examiner conducted all clinical examinations. The calibrated examiner was blinded to the treatment modality that has been provided to each subject.

The primary outcome was an assessment of plaque index (Silness and Loe 1964), GI (Loe and Silness 1963), BI (Van Der ouderaa 1989), PPD, and CAL at baseline for all the subjects. Plaque index, GI, and $\mathrm{BI}$ were assessed on four gingival areas of teeth: mesial, distal, buccal, and lingual surface for each tooth. The William's periodontal probe was used to measure the PPD, which was measured from the free gingival margin to the base of the pocket at the selected sites and the CAL was measured from cementoenamel junction to the base of the pocket.

The secondary outcome was the assessment of the same clinical parameters for all the subjects after the treatment at day 90 of the study.

\section{Examiner Calibration}

All measurements of clinical parameters were performed by the principal investigator and a calibrated examiner. At baseline, the 
clinical parameters were assessed by the principal investigator, whereas, at day 90, clinical parameters were assessed by the calibrated examiner who is not a part of the study to avoid bias.

\section{Statistical Analysis}

Data analysis was carried out using a statistical package for social science software (Version 22.0, SPSS ${ }^{\circledR}$ Inc., Chicago, IL, USA). The values of different parameters are expressed as mean standard deviation. A paired $t$ test was used for intragroup comparison. For intergroup comparisons, an unpaired $t$ test was used. The mean changes from baseline to day 90 were calculated for all the parameters. A $p$ value of $<0.05$ was considered as statistically significant, a $p$ value of $<0.002$ is considered as high statistical significant, and a $p$ value of $<0.001$ is considered as very high statistical significant. The level of significance in the present study was $5 \%$ and the power of the study was calculated to be $80 \%$.

\section{Results}

\section{Characteristics of the Study Population}

A total of 30 subjects with 60 teeth were randomly divided into two groups depending on the treatment provided and were statistically analyzed for the age of subjects, to evaluate the clinical effects of SRP in the treatment of subjects with CP, to evaluate the clinical effects of SRP along with $\mathrm{DL}$ as an adjunct in the treatment of subjects with $C P$, and to compare the effectiveness of $D L$ as an adjunct to SRP and SRP alone in the treatment of subjects with CP. In groups I and II, the mean value of age was $36.733 \pm 7.965$ which implied that there was no statistically significant difference between the groups.

\section{Clinical Parameters}

In the intragroup comparison, there was a decrease in the mean difference of $\mathrm{Pl}, \mathrm{Gl}$, and $\mathrm{BI}$ individually for both the groups from baseline to 3 months and the difference was statistically significant $(p<0.001)$. In the intergroup comparison (Table 1), $\mathrm{Pl}, \mathrm{Gl}$, and $\mathrm{BI}$ in both the groups were similar at baseline and 3 months, so there was no statistically significant difference $(p=1)$.

On comparison of mean values of PPD from baseline to 3 months between groups I and II on the mesial and distal aspect showed statistically significant difference (IM: $0.633 \pm 0.490$; IIM: $1.100 \pm 0.923, p=0.017$; ID: $0.667 \pm 0.711 ;$ IID: $1.467 \pm 0.937, p<0.01$; Table 2) whereas, on the buccal and palatal/lingual aspect, it was not statistically significant (IB: $0.333 \pm 0.606$; IIB: $0.600 \pm 0.724, p=$ $0.127 ;$ IP/L: $0.333 \pm 0.606$; IIP/L: $0.633 \pm 0.809, p=0.109$, Table 2). On intragroup comparison of mean values of PPD on all four aspects of the teeth from baseline to 3 months, there was a statistically significant difference with $p<0.001$ (Table 3 ).

On comparison of mean values of CAL from baseline to 3 months between groups I and II on distal and buccal aspect showed there was a statistical significant difference (ID: $0.733 \pm 0.583$; IID: $1.433 \pm 0.858, p<0.001$; IIB: $0.667 \pm 711, p=0.05$, Table 4) whereas, on the mesial and palatal/lingual aspect, it was not statistically significant (IM: $0.733 \pm 583$; IIM: $1.133 \pm 937, p=0.151$; IP/L: $0.333 \pm$ 661 ; IIP/L: $633 \pm 0.809, p=0.121$, Table 4). On intragroup comparison of mean values of CAL on all four aspects of the teeth from baseline to 3 months, there was a statistically significant difference with $p$ $<0.001$ (Table 5).

\section{Discussion}

Scaling and root planing is the primary recommended approach to control periodontal infection. However, the complete removal of bacterial biofilm and their endotoxins in deeper, inaccessible areas of the pockets and furcation sites is often difficult to achieve by performing SRP alone. So, this necessitated the use of additional therapeutic strategies as an adjunct to SRP.

The use of the laser as an adjunct to conventional SRP is based on the understanding that the lasers can cause good tissue ablation, with strong bactericidal and detoxification effects, and also can reach deeper sites which are inaccessible to the conventional mechanical instrumentation. Application of DL with a wavelength between 655 and $980 \mathrm{~nm}$ is known to accelerate wound healing

Table 1: Comparison of mean plaque index, gingival index, and bleeding index from baseline to 3 months between groups I and II

\begin{tabular}{llllll}
\hline Groups & & $N$ & Mean & Std. deviation & $T$ \\
\hline Plaque index & Group I & 30 & 0.455 & 0.313 & 0.000 \\
& Group II & 30 & 0.455 & 0.313 & $p=1 \mathrm{~ns}$ \\
Gingival index & Group I & 30 & 0.407 & 0.318 & 0.000 \\
& Group II & 30 & 0.407 & 0.318 & $p=1 \mathrm{~ns}$ \\
Bleeding index & Group I & 30 & 0.437 & 0.286 & 0.000 \\
& Group II & 30 & 0.437 & 0.286 & $p=1 \mathrm{~ns}$ \\
\hline
\end{tabular}

Table 2: Comparison of mean probing pocket depth from baseline to 3 months between groups I and II

\begin{tabular}{llllll}
\hline Groups & & $N$ & Mean & Std. deviation & $T$ \\
\hline PPD-M & Group I & 30 & 0.633 & 0.490 & 2.446 \\
& Group II & 30 & 1.100 & 0.923 & $p=0.017 \mathrm{sig}$ \\
PPD-D & Group I & 30 & 0.667 & 0.711 & 3.725 \\
& Group II & 30 & 1.467 & 0.937 & $p<0.001 \mathrm{sig}$ \\
PPD-B & Group I & 30 & 0.333 & 0.606 & 1.547 \\
& Group II & 30 & 0.600 & 0.724 & $p=0.127 \mathrm{~ns}$ \\
PPD-P/L & Group I & 30 & 0.333 & 0.606 & 1.626 \\
& Group II & 30 & 0.633 & 0.809 & $p=0.109 \mathrm{~ns}$ \\
\hline
\end{tabular}

PPD, probing pocket depth; $M$, mesial; $D$, distal; B, buccal; P/L, palatal/lingual 
Table 3: Comparison of mean probing pocket depth from baseline to 3 months in groups I and II

\begin{tabular}{llllll}
\hline \multirow{2}{*}{ Groups } & & \multicolumn{2}{c}{ Paired differences } & & \\
\cline { 3 - 5 } Group I & & Mean & Std. deviation & $T$ & $<$ Value \\
& PPD-M & 0.633 & 0.490 & 7.077 & $<0.001 \mathrm{sig}$ \\
& PPD-D & 0.667 & 0.711 & 5.135 & $<0.001 \mathrm{sig}$ \\
Group II & PPD-B & 0.333 & 0.606 & 3.010 & $0.005 \mathrm{sig}$ \\
& PPD-P/L & 0.333 & 0.606 & 3.010 & $0.005 \mathrm{sig}$ \\
& PPD-M & 1.100 & 0.923 & 6.528 & $<0.001 \mathrm{sig}$ \\
& PPD-D & 1.467 & 0.937 & 0.572 & $<0.001 \mathrm{sig}$ \\
\hline
\end{tabular}

PPD, probing pocket depth; $M$, mesial; $D$, distal; B, buccal; P/L, palatal/lingual

Table 4: Comparison of mean clinical attachment level from baseline to 3 months between groups I and II

\begin{tabular}{llllll}
\hline & Groups & $N$ & Mean & Std. deviation & $T$ \\
\hline CAL-M & Group I & 30 & 0.733 & 0.583 & 1.985 \\
& Group II & 30 & 1.133 & 0.937 & $p=0.151 \mathrm{~ns}$ \\
CAL-D & Group I & 30 & 0.667 & 0.758 & 3.667 \\
& Group II & 30 & 1.433 & 0.858 & $p<0.001 \mathrm{sig}$ \\
CAL-B & Group I & 30 & 0.333 & 0.606 & 1.990 \\
& Group II & 30 & 0.667 & 0.711 & $p=0.05 \mathrm{sig}$ \\
CAL-P/L & Group I & 30 & 0.333 & 0.661 & 1.573 \\
& Group II & 30 & 0.633 & 0.809 & $p=0.121 \mathrm{~ns}$ \\
\hline
\end{tabular}

CAL, clinical attachment level; M, mesial; D, distal; B, buccal; P/L, palatal/lingual

Table 5: Comparison of mean clinical attachment level from baseline to 3 months in groups I and II

\begin{tabular}{|c|c|c|c|c|c|}
\hline \multirow[b]{2}{*}{ Groups } & & \multicolumn{2}{|c|}{ Paired differences } & \multirow[b]{2}{*}{$T$} & \multirow[b]{2}{*}{$p$ Value } \\
\hline & & Mean & Std. deviation & & \\
\hline \multirow[t]{4}{*}{ Group I } & CAL-M & 0.733 & 0.583 & 6.886 & $<0.001 \mathrm{sig}$ \\
\hline & CAL-D & 0.667 & 0.758 & 4.817 & $<0.001$ sig \\
\hline & CAL-B & 0.333 & 0.606 & 3.010 & $0.005 \mathrm{sig}$ \\
\hline & CAL-P/L & 0.333 & 0.661 & 2.763 & $0.010 \mathrm{sig}$ \\
\hline \multirow[t]{4}{*}{ Group II } & CAL-M & 1.133 & 0.937 & 6.624 & $<0.001 \mathrm{sig}$ \\
\hline & CAL-D & 1.433 & 0.858 & 9.146 & $<0.001 \mathrm{sig}$ \\
\hline & CAL-B & 0.667 & 0.711 & 5.135 & $<0.001 \mathrm{sig}$ \\
\hline & CAL-P/L & 0.633 & 0.809 & 4.289 & $<0.001 \mathrm{sig}$ \\
\hline
\end{tabular}

CAL, clinical attachment level; $M$, mesial; $D$, distal; $B$, buccal; $P / L$, palatal/lingual

through the facilitation of formation of collagen, brings about angiogenesis and augmentation of growth factor. ${ }^{5}$ However, despite the potential benefits of the use of lasers in periodontology, there are limited clinical evidences that have confirmed the use of lasers as an adjunct to conventional periodontal therapy may provide additional benefits in the treatment of subjects with CP.

The present study aimed to compare the effectiveness of DL as an adjunct to SRP and SRP alone in the treatment of subjects with CP. The results indicate that SRP using hand instruments and an ultrasonic device alone (group I) or in combination with a DL (group II) provide significant improvements in clinical parameters like plaque index, GI, BI, PPD, and CAL in both groups I and II from baseline to 3 months, i.e., after the treatment of subjects with CP.

In the present study, there are no statistically significant improvement in plaque, gingival and bleeding indices from baseline to 3 months, with adjunctive use of $810 \mathrm{~nm} \mathrm{DL}$ with
SRP, this particular finding is in agreement with Assaf et al. ${ }^{7}$ who used the same specifications of DL irradiation which was used in our study. Although both groups I and II showed positive results after the treatment with very high statistical significance, group II demonstrated better improvements in terms of mean difference of PPD reduction on mesial and distal aspects in moderate pockets measuring 4-6 mm during baseline to 3 months. The mean difference in the gain in the CAL has shown very high statistically significant values on the distal side of the laser-treated teeth, however, the improvement was not statistically significant on the mesial side. This observation is in line with Dukić et al. ${ }^{8}$ study, where they found no improvement in the CAL at all. Thus, the possible explanation for the reduction in PPD could be the gingival tissue retraction rather than clinical attachment gain. But, there is a statistically significant improvement in CAL on the buccal aspect, this can be attributed to the de-epithelization of periodontal 
pockets leading to increased connective tissue attachment which is in agreement with the study conducted by Kreisler et al. ${ }^{9}$ and Kamma et al. ${ }^{10}$

Caruso et al. ${ }^{11}$ demonstrated that laser as an adjunct to SRP has a bactericidal effect, which was assessed by the collection of plaque samples using PCR. Thus, the elimination of bacteria from periodontal pockets promotes a considerable amount of healing of periodontal tissues.

De Micheli et al. ${ }^{12}$ conducted a similar study using a high power DL in combination with SRP performed on day 1 and 7. They did not get significant improvements in clinical parameters in contrast to our findings. The better results in our study could be due to the extended use of laser for 3 times (day 1, 7, and 21). Similarly, Ribeiro et al. ${ }^{13}$ did not observe differences in the measured clinical parameters between test and control sites, here results may differ because of the difference in the interval of laser irradiation which was done after 1, 2, and 3 days of SRP. Similarly, De Micheli et al. ${ }^{12}$ observed no difference in terms of PPD and CAL in sites irradiated with a laser at the end of the 6th month follow-up period.

In the present study, split-mouth design was followed, both groups I and II received SRP, and only group II received laser irradiation of $810 \mathrm{~nm}$, continuous wave mode, the output power of $2.5 \mathrm{~W}$, input (vol)-16.8 V, for 20 seconds in apico-coronal direction with optic fiber diameter being $400 \mu \mathrm{m}$ on days 7, 14, and 21 after SRP, whereas group I received no other treatment except SRP.

The above-mentioned specifications of laser irradiation were chosen based on the observations made in a systematic review conducted by Qadri et al. ${ }^{5}$ which suggests that the use of these laser power settings are sufficient to eliminate the periodontal pocket epithelium. The greater reduction of PPD in the current study could be attributable to the use of laser irradiation with these specifications.

The application of DLs on periodontal tissues has demonstrated the increase in the mRNA expression of insulin growth factorbeta and vascular endothelial growth factor in human gingival fibroblasts which demonstrates a potent effect on the metabolism of connective tissues and it also brings down the production of matrix metalloproteinase- 8 which plays an important role in the degradation of collagen fibers. ${ }^{5}$

The hemoglobin in the soft tissue of periodontal pockets acts as an absorber chromophore and as an endogenous dye for a high power $D L$, thereby enhancing the effect of the laser at the site of the application, which demonstrates the bactericidal effect of a DL. ${ }^{12}$

In the present study, the improvements in terms of clinical parameters have been demonstrated in groups I and II individually, this result is in agreement with a study conducted by Dukić et al. ${ }^{8}$ The possible explanation for this result is that SRP is known to stop the inflammatory processes by reducing the number of pathogenic periodontal microorganisms, which will also facilitate the removal of smear layer, bacterial endotoxins, and contaminated root cementum, which in turn facilitates healing of periodontal tissues in shallow to moderate periodontal pockets but not in deeper pockets which necessitates further adjunctive use of various other treatment modalities.

To the best of our knowledge, this is the first study to observe the differences in PPD and CAL in site-specific manner rather than considering the total average value of PPD and CAL around each tooth. Following this pattern of assessment of PPD and CAL has rendered us more accuracy in comparing the potential benefits of each treatment modality that has been provided in this study.
Other important observations that were made in this study are:

- Maintenance of good oral hygiene potentiated the treatment modalities that have been provided in the course of the study.

- The compliance of the patients becomes an important and integral part of any treatment plan and outcome. In this regard, better compliance of patients can be obtained if the treatment protocol is explained in their language.

However, there was no statistically significant difference between groups I and II in terms of PPD and CAL in buccal, palatal/ lingual aspects, and mesial aspects, palatal/lingual aspects, respectively. De Micheli et al. ${ }^{12}$ suggested the use of power meter while working with lasers to assess and visualize the parametric values which quantify the actual amount of energy that is present at the tip of the fiber optic delivery system and how much energy is lost when the laser beam is transmitted through fiber optic. Dukić et al. ${ }^{8}$ opined that the literature does not show any standard procedures regarding energy, group distribution, modes of irradiation, and time taken for a bacterial reduction in the periodontal pocket, which hinders the precise analysis of the results.

The study will have a broader scope if we include the microbial assessment; periodontal pocket with varying depths and extend the study for a longer period of time.

The results of the study demonstrated that, compared with SRP alone, adjunctive use of $810 \mathrm{~nm}$ DL with SRP offers a good additional benefit in the treatment of subjects with CP. According to a precise site-specific analysis of results, the adjunctive use of $810 \mathrm{~nm}$ DL to conventional SRP showed moderate improvement in terms of PPD and CAL. Furthermore, additional longitudinal, randomized, microbial, and controlled clinical trials with larger sample size and laser irradiation for periodontal pocket measuring $>6 \mathrm{~mm}$ are necessary to adequately test the potential benefits of DL-mediated periodontal therapy and it is also necessary to provide standard criteria for periodontal laser therapy in terms of laser power settings and mode of irradiation which would facilitate the analysis of the obtained results.

\section{Conclusion}

Within the limits of the present study, it can be concluded that the adjunctive use of $810 \mathrm{~nm}$ DL with SRP provides moderate additional clinical benefit in moderate periodontal pockets $4-6 \mathrm{~mm}$.

\section{Clinical Significance}

This study signifies that using noninvasive laser therapy, potential clinical benefits can be obtained in terms of reduction of periodontal parameters.

\section{References}

1. Saini R, Marawar PP, Shete $S$, et al. Periodontitis, a true infection. J Glob Infect Dis 2009;1(2):149-150. DOI: 10.4103/0974-777X.56251.

2. Taba M, Kinney J, Kim SA, et al. Diagnostic biomarkers for oral and periodontal diseases. Dent Clin North Am 2005;49(3):551-571. DOI: 10.1016/j.cden.2005.03.009.

3. Mittal A, Nichani AS, Venugopal RV. The effect of various ultrasonic and hand instruments on the root surfaces of human single rooted teeth: a planimetric and profilometric study. J Indian Soc Periodontol 2014;18(6):710-717. DOI: 10.4103/0972-124X.147405.

4. Sagar K, Kaur A, Patel P, et al. Diode laser as an established tool in periodontics - a review. Am J Oral Med Radiol 2015;2(2):54-60. 
5. Qadri T, Javed F, Johannsen $G$, et al. Role of diode lasers (800-980 $\mathrm{nM}$ ) as adjuncts to scaling and root planing in the treatment of chronic periodontitis: a systematic review. Photomed Laser Surg 2015;33(11):568-575. DOI: 10.1089/pho.2015.3914.

6. Bonito AJ, Lux L, Lohr KN. Impact of local adjuncts to scaling and root planing in periodontal disease therapy: a systematic review. J Periodontol 2006;77(2):326-327. DOI: 10.1902/jop.2006.620003.

7. Assaf M, Yilmaz S, Kuru B, et al. Effect of the diode laser on bacteremia associated with dental ultrasonic scaling: a clinical and microbiological study. Photomed Laser Surg 2007;25(4):250-256. DOI: 10.1089/pho.2006.2067.

8. Dukić W, Bago I, Aurer A, et al. Clinical effectiveness of diode laser therapy as an adjunct to non-surgical periodontal treatment: a randomized clinical study. J Periodontol 2013;84(8):1111-1117. DOI: 10.1902/jop.2012.110708.

9. Kreisler M, Al Haj H, d'Hoedt B. Clinical efficacy of semiconductor laser application as an adjunct to conventional scaling and root planing. Lasers Surg Med 2005;37(5):350-355. DOI: 10.1002/Ism. 20252.

10. Kamma JJ, Vasdekis VG, Romanos GE. The effect of diode laser (980 $\mathrm{nM}$ ) treatment on aggressive periodontitis: evaluation of microbial and clinical parameters. Photomed Laser Surg 2009;27(1):11-19. DOI: 10.1089/pho.2007.2233.

11. Caruso U, Nastri L, Piccolomini R, et al. Use of diode laser $980 \mathrm{nM}$ as adjunctive therapy in the treatment of chronic periodontitis A randomized controlled clinical trial. New Microbiol 2008;31(4): 513-518.

12. De Micheli G, De Andrade AK, Alves VT, et al. Efficacy of high intensity diode laser as an adjunct to non-surgical periodontal treatment: a randomized controlled trial. Lasers Med Sci 2011;26(1):434-438.

13. Ribeiro IW, Sbrana MC, Esper LA, et al. Evaluation of the effect of the GaAlAs laser on subgingival scaling and root planing. Photomed Laser Surg 2008;26(4):387-391. DOI: 10.1089/pho.2007. 2152. 